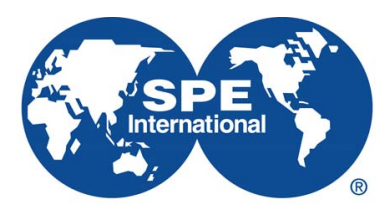

Society of Petroleum Engineers

\title{
SPE-190398-MS
}

\section{Foam Generation with Flow Rate: Effect of Surfactant Concentration and Gas Fraction}

\author{
G. Yu and W. R. Rossen, Delft University of Technology; S. Vincent-Bonnieu, Shell Global Solutions \\ International B. V. \\ Copyright 2018, Society of Petroleum Engineers \\ This paper was prepared for presentation at the SPE EOR Conference at Oil and Gas West Asia held in Muscat, Oman, 26-28 March 2018.
}

This paper was selected for presentation by an SPE program committee following review of information contained in an abstract submitted by the author(s). Contents of the paper have not been reviewed by the Society of Petroleum Engineers and are subject to correction by the author(s). The material does not necessarily reflect any position of the Society of Petroleum Engineers, its officers, or members. Electronic reproduction, distribution, or storage of any part of this paper without the written consent of the Society of Petroleum Engineers is prohibited. Permission to reproduce in print is restricted to an abstract of not more than 300 words; illustrations may not be copied. The abstract must contain conspicuous acknowledgment of SPE copyright.

\section{Abstract}

The propagation of foam in an oil reservoir depends on the creation and stability of the foam in the reservoir, specifically the creation and stability of foam films, or lamellae. As the foam propagates far from in injection well, superficial velocity and pressure gradient decrease with distance from the well. Experimental (Friedmann et al., 1994) and theoretical (Ashoori et al., 2011) studies relate concerns about foam propagation at low superficial velocity to the minimum velocity for foam generation near the well (Rossen and Gauglitz, 1990; Gauglitz et al., 2002). The objective of this work is to measure the impact of surfactant concentration and gas fractional flow on foam generation. Theory (Rossen and Gauglitz, 1990; Kam and Rossen, 2003) relates foam generation to gas fractional flow and, indirectly, to the stability of foam films, or lamellae, which in turn depends on surfactant concentration (Apaydin and Kovcsek, 2001). However, the link between foam generation and surfactant concentration has not been established experimentally.

In our experiments, nitrogen foam is generated in a core of Bentheimer sandstone. The foamgeneration experiments consist of measuring the critical velocity for foam generation as a function of gas fractional flow at three surfactant concentrations well above the critical micelle concentration. Experimental results show that critical velocity decreases with increasing liquid fraction, as shown by previous foam generation studies (Rossen and Gauglitz, 1990; Friedmann et al., 1991). Additionally, our results show that the critical velocity decreases with increasing surfactant concentration, far above the CMC. We also propose a workflow for screening out the experimental artifacts that can distort the trigger velocity.

\section{Introduction}

Gas-injection enhanced oil recovery (EOR) can efficiently displace oil (Moritis, 1990; Rossen, 1996; Lake et al., 2014). However, gas-injection EOR suffers from poor sweep efficiency and may achieve limited oil recoveries in field applications (Rossen, 1996; Lake et al., 2014), primarily due to low gas viscosity (leading to fingering and channelling), low gas density (leading to gravity override) and geological heterogeneity. Reducing the relative mobility of gas thus becomes a major challenge for gasinjection EOR. Foam can provide mobility control for gas flooding. Foam is a dispersion of gas bubbles in an aqueous phase, stabilized 
by surfactant molecules at the gas-liquid interfaces. When foam is generated in porous media, the flow paths of gas are blocked by liquid films, or lamellae, while the liquid phase remains continuous. The lamellae blocking the gas phase add additional capillary resistance to gas flow and thereby make the gas phase less mobile.

The conditions for foam generation depend in part on the method of injection. In our experiments, we consider steady gas and liquid injection at a fixed gas fraction, where gas has already been injected for a time before surfactant is added to the system (Rossen and Gauglitz, 1990). This initial state is relevant to the propagation of a foam front far from a well, where alternating slugs of gas and liquid have mixed and where gas has advanced ahead of the foam front. During steady-state experiments, foam is created in the porous medium by co-injecting gas and surfactant solution at a fixed gas fraction; foam generation requires exceeding a minimum superficial velocity $\mathrm{u}_{\mathrm{t}}{ }^{\mathrm{min}}$, or pressure gradient $\nabla_{\mathrm{P}}{ }^{\mathrm{min}}$ (Rossen and Gauglitz, 1990). "Foam generation", in this context, refers to an abrupt jump from a state of high gas mobility to one of very low mobility. This abrupt change depends on the rate of lamella creation exceeding the rate of lamella destruction in the porespace (Falls et al., 1988; Kovscek and Radke, 1996), leading to a spontaneous runaway process and a jump in state (Kam and Rossen, 2003; Kam, 2008). In this paper, we refer to this critical pressure gradient or superficial velocity as the 'trigger' for foam generation.

The triggers $\mathrm{u}_{\mathrm{t}}^{\min }$ or $\nabla_{\mathrm{p}}$ min depend on gas fractional flow (foam quality $\mathrm{f}_{\mathrm{g}}$ ). Greater $\mathrm{f}_{\mathrm{g}}$ requires a greater velocity to trigger foam generation (Rossen and Gauglitz, 1990). In the vicinity of an injection well, insitu foam generation and foam propagation is usually easy due to large superficial velocity and pressure gradient. The real concern for generation and propagation, therefore, lies in locations far from the injection well, where both superficial velocity and pressure gradient are low (Friedmann et al., 1994; Ashoori et al., 2011). Hence, the critical velocity for foam generation and propagation in porous media is of great importance to foam application.

Previous experimental studies haven't identified a connection between the minimum velocity for foam generation and surfactant concentration. The mechanisms of individual lamella generation (leavebehind, snap-off, lamella mobilization) are not believed to depend on the presence of surfactant (Ransohoff and Radke, 1988; Gauglitz and Radke, 1989; Kovscek et al., 1995). The survival of lamellae once created, however, does depend on surfactant formulation and concentration (Rossen, 1996). Foam generation requires not only production of lamellae in the porous medium, but also the survival of the newly created lamellae. The greater the lamella-destruction rate (either due to ineffective surfactant or insufficient surfactant concentration), the greater the lamella-creation rate needed to generate foam. The stability of foam in porous media, reflected in the limiting capillary pressure $\mathrm{P}_{\mathrm{c}}{ }^{*}$ or water saturation $\mathrm{S}_{\mathrm{w}}{ }^{*}$ for foam stability, increases with increasing surfactant concentration far above the Critical Micelle Concentration (CMC) (Apaydin and Kovscek, 2001; Jones et al., 2016) Therefore, one would expect that increasing surfactant concentration reduces the threshold superficial velocity or pressure gradient for triggering foam generation by reducing the rate of lamella breakage. However, this link has not been demonstrated experimentally.

In this paper we present experimental verification of the connection between the minimum velocity for foam generation and surfactant concentration for one surfactant formulation. We also propose a workflow for identifying the triggering velocity and screening out the experimental artifacts. We relate the experimental results to a model for the trigger for foam generation. The model agrees with the trends of the experimental results.

\section{Experiments on foam generation}

\section{Experimental method and materials}

In our experiments, foam is generated in-situ by co-injecting surfactant solution and nitrogen into a homogeneous Bentheimer sandstone core at a back-pressure of 40 bar and a temperature of $30^{\circ} \mathrm{C}$. The main 
objective of our experiments is to map out the minimum superficial velocity $u_{t}^{\text {min }}$ required to trigger foam generation for different foam qualities $f_{g}$ and three surfactant concentrations $C_{s}$, each far above the CMC. Based on the measurement of the CMC by Jones et al. (2016), all three surfactant concentrations are far above the CMC, which is approximately $0.005 \mathrm{wt} \%$ for $\mathrm{AOS}$ with $3.0 \mathrm{wt} \% \mathrm{NaCl}$.

We use the same surfactant, Sodium C14-16 Alpha Olefin Sulfonate (AOS-1, Bioterge AS-40), for all experiments. Both brine and surfactant solutions contained $3 \mathrm{wt} \% \mathrm{NaCl}$. Figure 1 shows the experimental apparatus. The Bentheimer core is $17 \mathrm{~cm}$ in length, with a diameter of $1 \mathrm{~cm}$. The permeability of the core is $1.87 \times 10^{-12} \mathrm{~m}^{2}$. Four absolute-pressure transducers are located along the core. Two of them are located on the inlet and outlet lines, respectively, while the other two are in direct contact with the core. The core is thus divided into three sections, with inlet and outlet sections $5.25 \mathrm{~cm}$ long, and the middle section $6.5 \mathrm{~cm}$ long (Figure 1). Three different surfactant concentrations are tested for impact on foam generation: $0.1 \mathrm{wt} \%, 0.3$ $\mathrm{wt} \%$ and $0.5 \mathrm{wt} \%$. Surface tensions of the three surfactant solutions are shown in Table A 2 in the Appendix.

A small pressure cell of volume $150 \mathrm{ml}$ lies between the core and the back-pressure regulator (BPR) to mitigate any fluctuations at the BPR. Since, as mentioned above, pressure gradient is thought to play an essential role in foam generation, any sudden increase or decrease in back-pressure would lead to an abrupt change in pressure gradient at the outlet of the core. In such cases, foam generation could be triggered near the outlet.
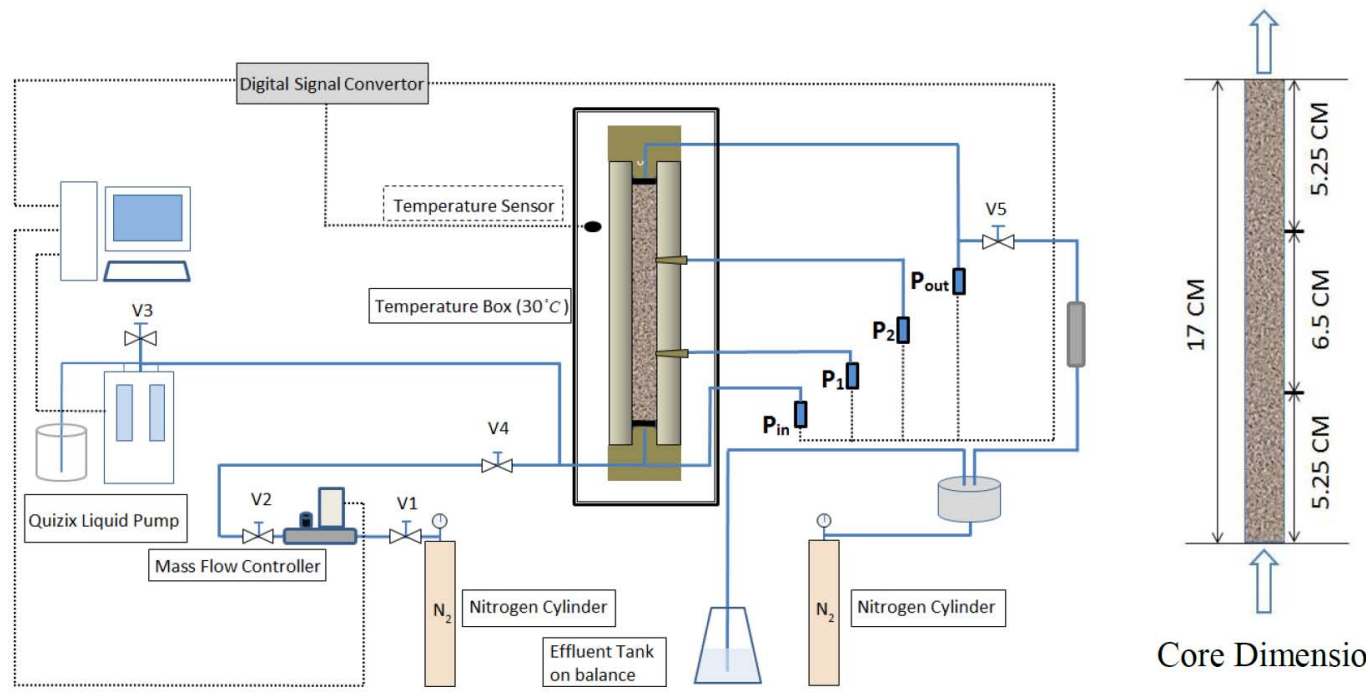

Core Dimension

\begin{abstract}
Figure 1-Experimental apparatus for foam-generation experiments. The core is mounted vertically in an oven at a temperature of $30^{\circ} \mathrm{C}$. Four absolute-pressure meters are connected along the core, with pressure ranges of 120 bar. Gas and liquid are injected from the bottom and exit from the top. A small metal container is connected between the last pressure meter $P_{\text {out }}$ and the back-pressure regulator to stabilize pressure in the outlet section of the core.
\end{abstract}

The core is initially fully saturated with brine. Then $\mathrm{N}_{2}$ and brine are co-injected at constant gas fractional flow. After steady state is achieved, brine injection is replaced by injection of surfactant solution at the same injection rate and fractional flow of gas. After 1 pore volume of surfactant solution has been injected, we begin the process of raising superficial velocity in steps until foam generation is triggered. At each step, we wait for a time to see if foam generation has occurred; details are given below. The trigger for foam generation could lie between the measured trigger velocity and the velocity just before it. The resulting uncertainty range for each experiment is illustrated by the error bars in the results shown below.

\title{
Experimental artifacts and screening criteria
}

Our goal is to determine the velocity at which foam generation occurs in steady flow in a homogeneous porous medium. Identification of the foam trigger (with regard to either velocity or pressure gradient) can 
be problematic, and experimental results are typically scattered, as illustrated in Figure 2. There are at least two experimental artifacts that contribute to the scatter: 1) the "incubation effect", and 2) the capillary end effect. Both effects may lead to foam generation at superficial velocities lower than the trigger velocity $u_{t}^{\min }$. These two effects are described below.

Baghdikian and Handy (1991), injecting liquid and gas into cores at steady, low velocities, observed a slow increase in $\nabla P$ until, many hours or even days later, there was an abrupt increase in $\nabla P$ over a period of minutes or hours: that is, "foam generation". They call this foam generation after a delay the "incubation effect" (see also Huh and Handy, 1989; Chou, 1991; Rossen, 1996). The reason for this behaviour is not clear, but it is likely the result of an accumulation of local perturbations in flow rates, foam quality, and capillary pressure, etc. over time, leading to creation of static lamellae and increasing pressure gradient (Rossen, 1996). We exclude these cases from our results, because we want to identify the point where velocity or pressure gradient triggers foam generation without the effects of extraneous fluctuations accumulated over time.

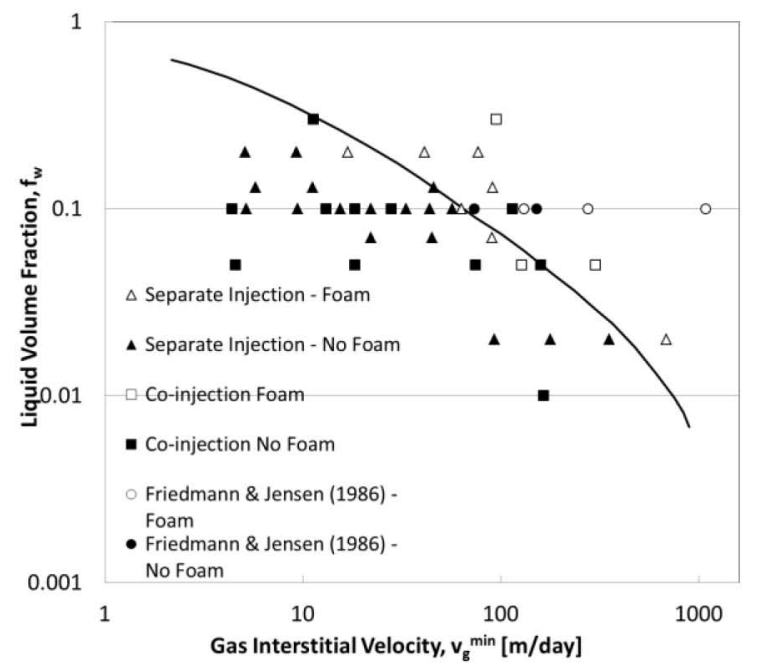

(a)

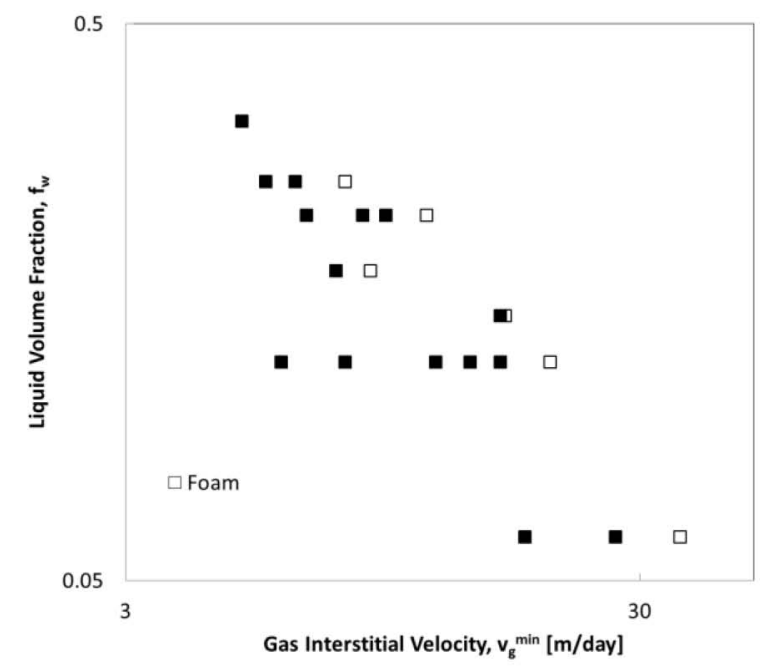

(b)

Figure 2-(a) Minimum gas interstitial velocity required to trigger foam generation as a function of injected liquid volume fraction (or $f_{w}$, i.e., $\left(1-f_{g}\right)$ ). The plot is reproduced from data of Rossen and Gauglitz (1990). Trends superimposed on data are from a percolationtheory analysis for foam generation described in Rossen and Gauglitz (1990). (b) A similar plot based on data from our experiments $\left(C_{s}=0.5 \mathrm{wt} \%\right)$. White dots represent the observed trigger velocity for the given injected liquid volume fraction, and black dots represent the velocities tested before the trigger of foam generation.

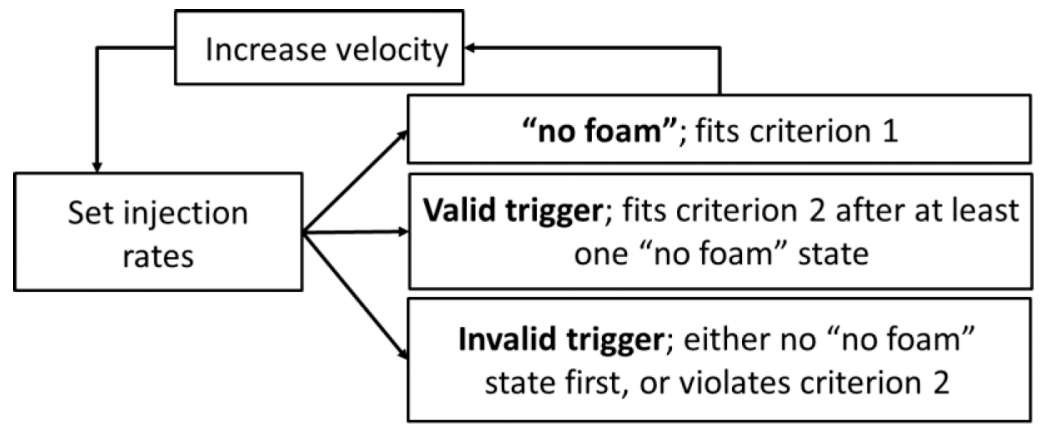

Figure 3-Experimental procedures for identification of a valid trigger velocity. Each experiment should begin at a superficial velocity lower than the trigger velocity. Three possible scenarios could happen at a particular velocity. (1) If no foam is created at this velocity (criterion 1), then a stepwise increase of superficial velocity is required, until a valid trigger, at which foam generation kicks off, is identified. (2) If foam generation takes place (meeting all conditions specified in criterion 2) after at least one "no foam" state, then a valid trigger velocity is identified. (3) If foam generation takes place at the very first "no foam" state, or any event(s) that violate criterion 2 take place during the process of velocity increase, the experiment is be aborted and repeated, until it meets both criteria and a valid trigger is identified. 
The capillary end effect (Perkins, 1957; Douglas et al., 1958; Kyte and Rapoport, 1958) is another complicating artefact in foam-generation experiments. Apaydin and Kovscek (2001) studied the role of surfactant concentration and end effects on foam flow in porous media. The classic capillary end effect is an accumulation of water near the outlet face of the porous medium caused by contact with fluid outside the porous medium with capillary pressure zero or near zero. The wet conditions near the core outlet are ideal for foam generation (Ransohoff and Radke, 1988). At larger surfactant concentrations, Apaydin and Kovscek (2001) reported, the end effect results in a larger pressure gradient building first near the outlet and propagating upstream, against the direction of flow, toward the inlet. Similar effects where a large increase in pressure gradient first occurs near the outlet and then propagates upstream during steady injection are reported by Nguyen et al. (2003) and Simjoo et al. (2013). The mechanism of upstream propagation of a stronger foam state is unclear, but, in any case, the origin of the state is a result of the capillary end effect, and therefore it is not representative of a homogeneous porous medium. Hence, we exclude cases in which a large pressure gradient is created near the outlet and then propagates to or disturbs upstream core sections.

We define the trigger as the total superficial velocity at which foam is created quickly near the core inlet, without a long period of steady injection or propagation of foam first created near the outlet. Below we define the criteria to define a valid trigger velocity and to identify unacceptable cases. Figure 3 illustrates how we identify a valid trigger according to two criteria:

1. The experiment should begin with at least one velocity lower than the trigger velocity for foam generation. In Figure 3 we call this state "no foam" for simplicity. In reality, it could be a state with a modest reduction of gas mobility, or what Ransohoff and Radke (1988) refer to as a "leave-behind foam." At this velocity, there should be no significant pressure drop in any core section. There are two criteria to define the condition before the trigger:

a. Pressure gradient along the entire core increases within the next 10-20 sec upon the increase of superficial velocity, and settles down to a new steady state quickly (usually within 20-30 sec). When the new steady state is achieved, the increase in pressure drop is of the same magnitude as the proportional increase in velocity from the previous step. Ideally this rule applies to all core sections. In many cases, however, the $\Delta \mathrm{P}$ across the outlet section increases much more than proportionately with the velocity increase, and more than the pressure drop in other sections. We accept cases with a modest $\Delta \mathrm{P}$ in the outlet section (no more than $1 \mathrm{bar}$, too little to affect gas volume or superficial velocity upstream) if the state of large $\nabla P$ doesn't migrate upstream to the second section. In other words, if there is foam generation near the outlet but this is not the cause of subsequent foam generation near the inlet, we accept that case.

b. Pressure gradient along the core should remain constant, without an upward trend, once a steady state is achieved. The period during which a steady pressure gradient is verified should be limited to avoid the "incubation effect" (see criterion 2a, below). We checked the steadystate of an injection rate for about 15 to $20 \mathrm{~min}$, before raising injection rate to the next level. If the injection period lasts for more than 40-60 min, the incubation effect could compromise the validity of result.

2. The trigger should be characterized by a rapid increase in pressure drop in all sections while keeping injection rate and foam quality constant. Specifically

a. The pressure drop across the first section rises steeply in the first section within 2 to 5 min of the increase in injection rate. The zone of large pressure gradient propagates from the first section downstream, but not from the last section upstream. A pressure rise occurring after, say, an hour of injection at a given rate could be a symptom of the incubation effect and unreliable.

b. At the trigger, the magnitude of increase in $\Delta \mathrm{P}$ is larger, and the period to reach the new steady state is longer (20-40 min), than in the steps before the trigger. The magnitude of gradient of the 
newly formed steady-state should be substantially greater (10 to 100 times) than the pressure gradient before the trigger.

If and only if both criteria are satisfied in our experiment, we identify a trigger velocity for the given surfactant concentration and foam quality. We denote this total superficial velocity as $\mathrm{u}_{\mathrm{t}, \mathrm{c}}$ below. If any of the above criteria are violated, the result of this experiment is discarded. The experiment should be repeated until a valid trigger is identified. Figure 4 shows examples of both valid (Figure 4a) and invalid (Figure 4b) experimental results.
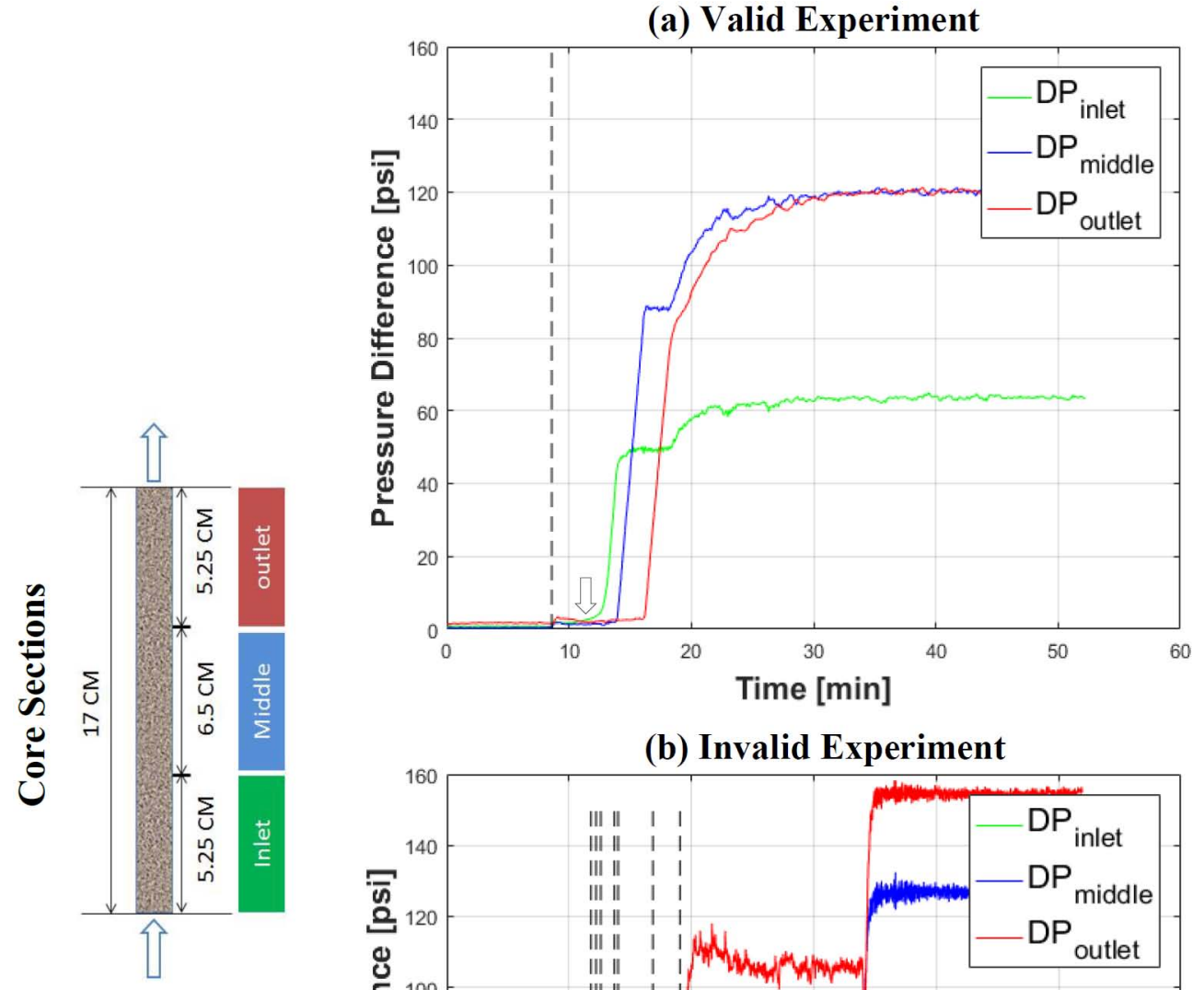

(b) Invalid Experiment

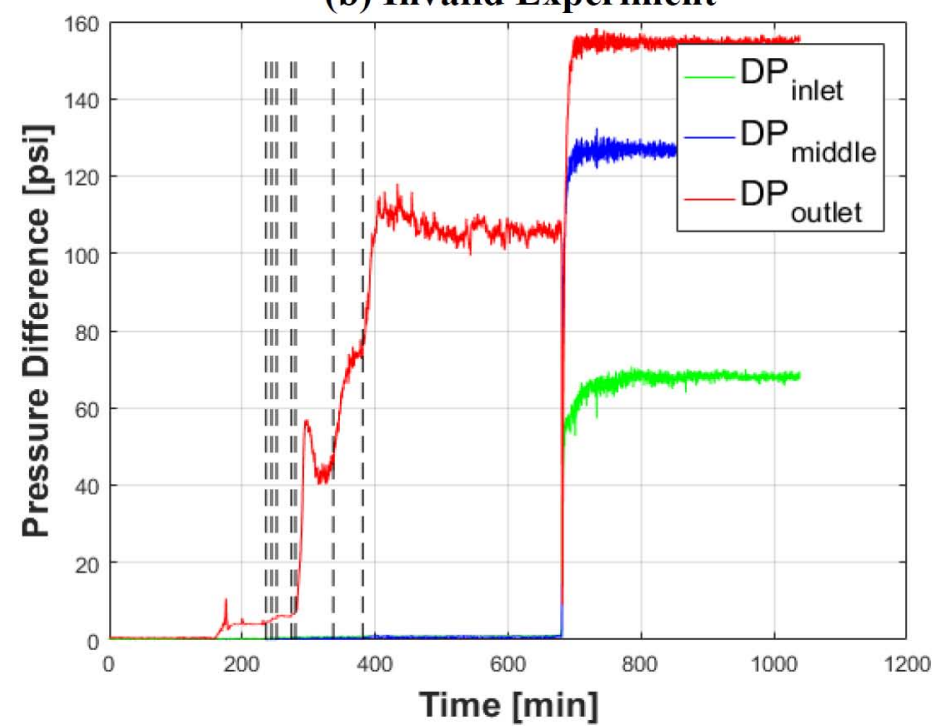

Figure 4-(a) A valid finding of a trigger velocity $\left(C_{s}=0.3 w t \%, f_{g}=85.04 \%\right)$. Upon the increase in injection rate at after about $81 / 2 \mathrm{~min}$. co-injection of surfactant solution and nitrogen, foam generation is triggered in the inlet section within $5 \mathrm{~min}$. and propagates downstream. (b) An invalid result $\left(C_{s}=0.3 \mathrm{wt} \%, f_{g}=87.98 \%\right)$. Weak foam is first created in the outlet section (at around $160 \mathrm{~min}$.) instead of upstream sections, likely due to end effect instead of velocity increase. Strong foam is created later near the outlet after a long period of injection (around $7 \mathrm{hr}$ ), and eventually pressure drop in the last section (110 psi) is large enough to affect superficial velocities upstream. Foam finally fills the core after about $700 \mathrm{~min}(12 \mathrm{hr})$, but the effect of the last section cannot be ruled out. 


\section{Results}

Our experiments (Figures 5, 6, and 8) show that 1) the critical superficial velocity $\mathrm{u}_{\mathrm{t}, \mathrm{c}}$ required to trigger foam generation increases with decreasing liquid fractional flow $f_{w}$, and 2) $u_{t, c}$ decreases with increasing surfactant concentration in the aqueous phase. Foam generation becomes easier for wetter foam (greater $\left.f_{w}\right)$ and higher surfactant concentration, even far above the CMC. The trend on this log-log plot (Figure 5) is roughly linear for each surfactant concentration. There is some scatter in the data, as in Figure 2, and some overlap between the data at some surfactant concentrations.

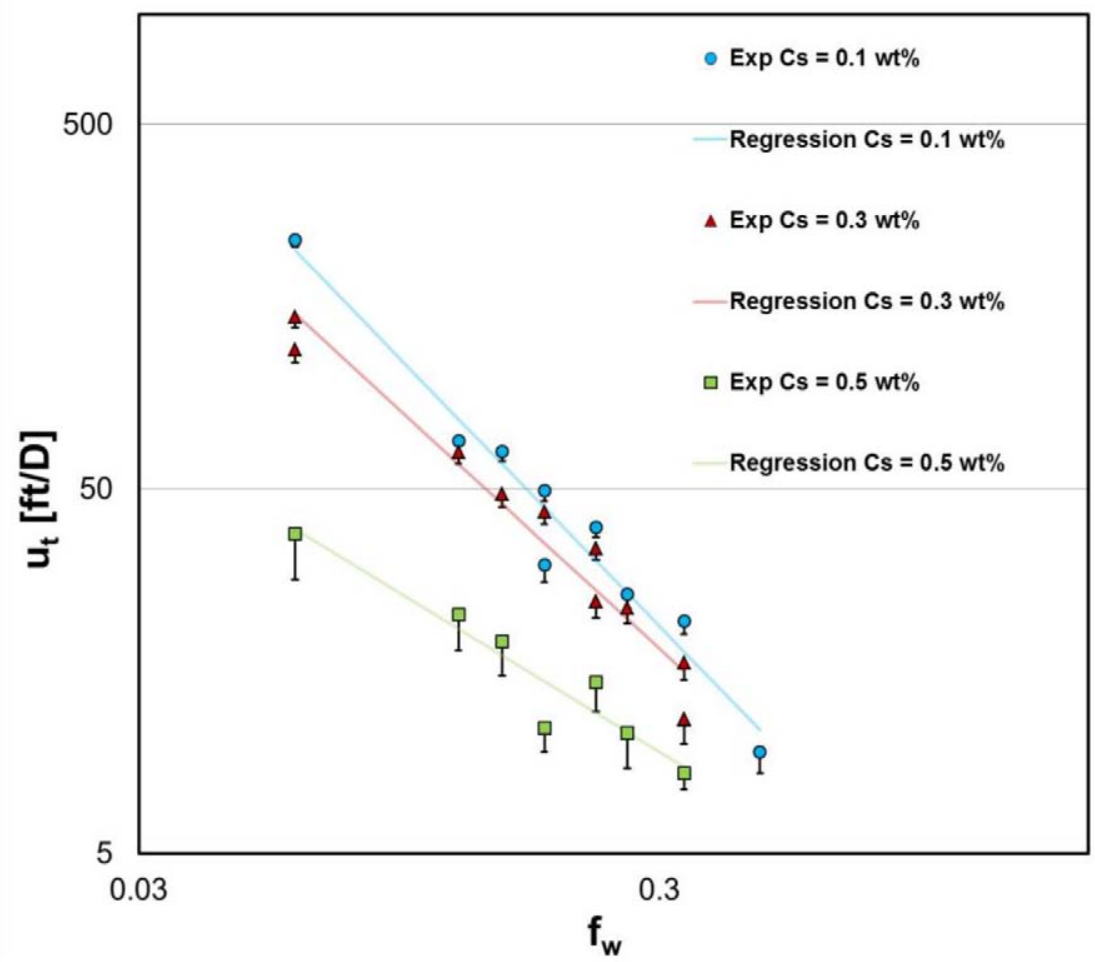

Figure 5-Experimental results for the trigger velocity for foam generation versus liquid fractional flow $f_{w}$ for three different surfactant concentrations. Data plotted on log-log scale approximate a linear trend (solid lines) for each surfactant concentration; the least-squares fit to each trend is also shown. The error bars (below data points) represent the difference between the trigger velocity and the velocity tested immediately before it. 


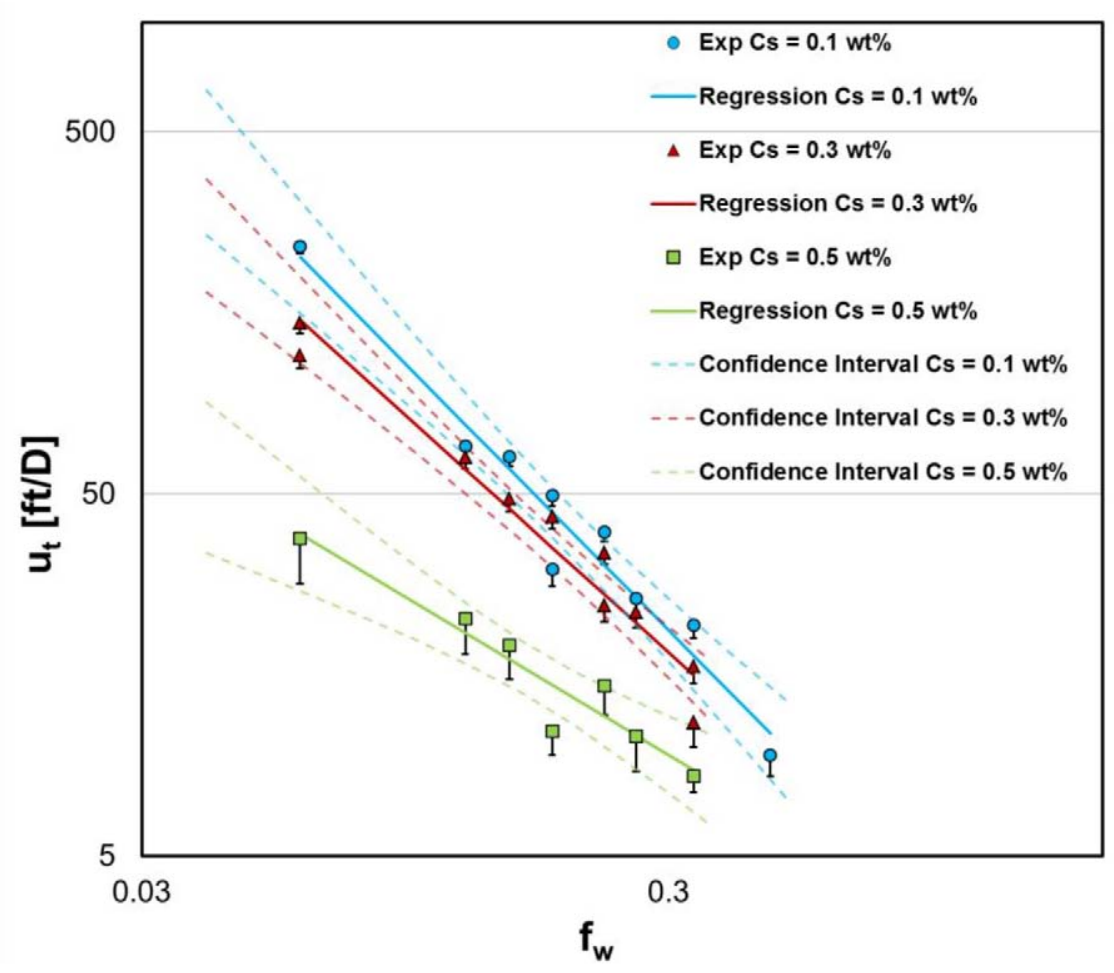

Figure 6-Estimated linear regression lines (solid lines) and $95 \%$ confidence intervals (dashed curves) for the underlying trends of the three surfactant concentrations. Markers represent the experimental results, as in Figure 5.

Figure 6 shows the regression lines as well as the 95\% confidence intervals for the trends (Wonnacott and Wonnacott, 1972) for the three surfactant concentrations used in our experiments. Although there is some overlap between the data for different surfactant concentrations, there is relatively little overlap between the confidence intervals for the trends at 0.1 and $0.3 \mathrm{wt} \%$ concentrations. There is no overlap between the top two trends and that at the bottom for $0.5 \mathrm{wt} \%$ concentration. In summary, surfactant concentration has an effect on foam generation that transcends the scatter in the individual data.

\section{Modeling the foam trigger}

Kam's population-balance model (Kam and Rossen, 2003) and its variants (Kam and Rossen, 2004; Kam, 2008) is the only population-balance model that explains the trigger for foam generation as seen in experiments (Gauglitz et al., 2002): i.e., that foam generation requires a minimum pressure gradient $\nabla_{\mathrm{P}}$ min , or, equivalently a minimum superficial velocity $\mathrm{u}_{\mathrm{t}}{ }^{\min }$. Like other population-balance models, this model represents foam texture explicitly, with rates of lamella creation and lamella coalescence defined by two functions. In this model, the rate of lamella creation depends on pressure gradient. Similar to other population-balance models, the rate of lamella destruction is controlled in this model by water saturation and the limiting water saturation $\mathrm{S}_{\mathrm{w}}{ }^{*}$, a parameter related to the limiting capillary pressure for foam destruction, $\mathrm{P}_{\mathrm{c}}{ }^{*}$ via the capillary-pressure/saturation function $\mathrm{P}_{\mathrm{c}}\left(\mathrm{S}_{\mathrm{w}}\right)$ (Khatib et al., 1988; Zhou and Rossen, 1995; Apaydin and Kovscek, 2001; Ma et al., 2013). As noted above, the process of lamella creation is not believed to depend on surfactant concentration; this assumption is incorporated into various populationbalance models (Friedmann et al., 1991; Kovscek et al., 1995; Kam and Rossen, 2003). $\mathrm{S}_{\mathrm{w}}{ }^{*}$ and $\mathrm{P}_{\mathrm{c}}{ }^{*}$ do depend on surfactant concentration far above the CMC (Apaydin and Kovscek, 2001; Jones et al., 2016). 


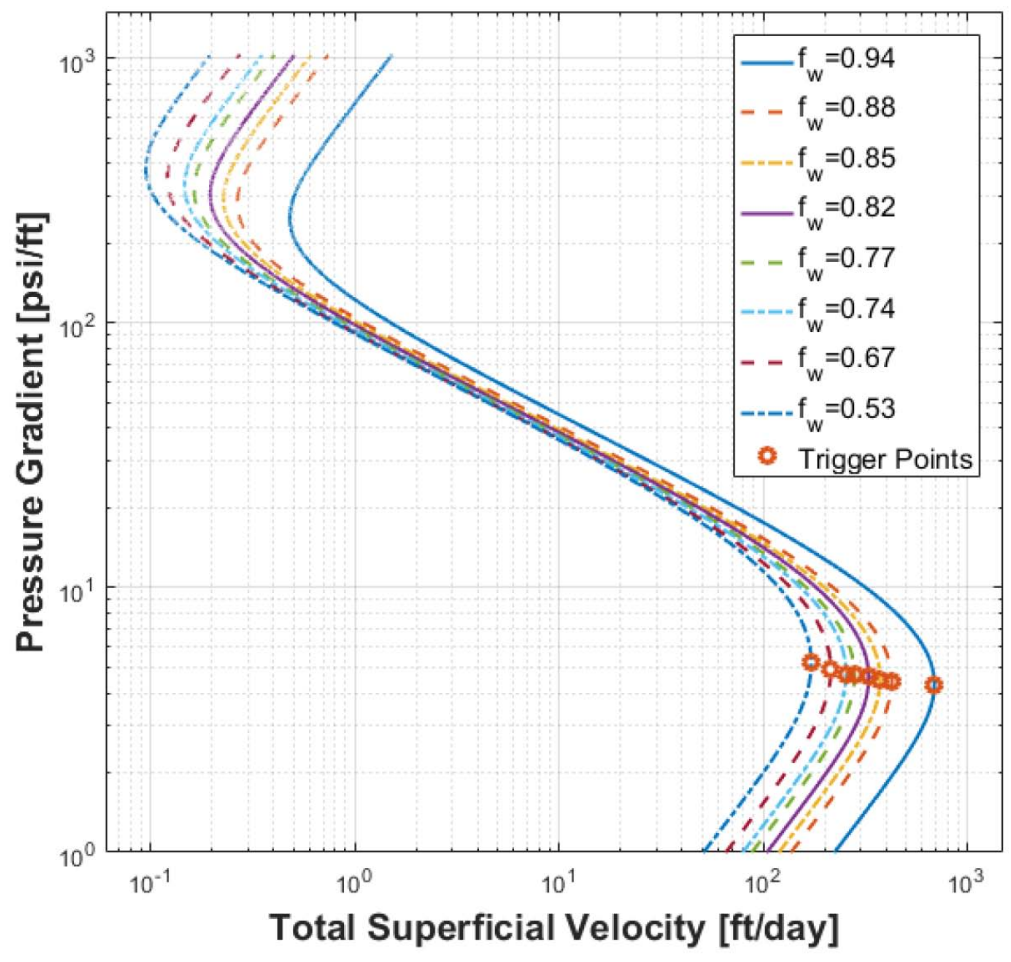

Figure 7-Steady-state interstitial velocity as function of pressure gradient for given foam qualities $f_{g}$, from Kam's population-balance model (Kam and Rossen, 2003) with parameters from Appendix A (specifically, $S_{w}{ }^{*}=0.201, S_{w c}=$ 0.2). The lower branch represents the steady state of weak foam (or no foam); the upper branch represents the steady state of strong foam. The trigger for foam generation is the maximum of the lower branch (orange circles), where the foam-generation function bends back to lower superficial velocities. These maximum values produce the blue curve in Figure 8, representing the trend for one value of $S_{w}{ }^{*}$, and hence $C_{s}$. In an experiment at fixed superficial velocity, there would be a jump from the weak/no-foam state to the strong-foam state at the maximum of the lower branch.

Figure 7 shows the relationship between pressure gradient and superficial velocity predicted by the model for one value of $\mathrm{S}_{\mathrm{w}}{ }^{*}$. The trigger for foam generation is the maximum velocity on the lower (weak-foam) branch, where the function bends back toward lower values of superficial velocity. The values of $f_{w}$ and $\mathrm{u}_{\mathrm{t}}$ at this maximum represent the relation between foam quality and critical velocity for foam generation for one value of $\mathrm{S}_{\mathrm{w}}{ }^{*}$. Figure 8 shows how the trend shifts with $\mathrm{S}_{\mathrm{w}}{ }^{*}$ and, by implication, with surfactant concentration.

The trend in superficial velocity $\mathrm{u}_{\mathrm{t}}$ against pressure gradient $\nabla \mathrm{P}$ predicted by Kam's model (Figure 8 ) is similar to the experimental results in Figures 5 and 6. The model parameters in Appendix A were fit to data for a different foam formulation in a different porous medium. We present the model results with this set of parameters merely to indicate the trend predicted by the model. A quantitative fit would require fitting all the parameters, possibly tweaking the functional forms used to represent lamella creation as a function of $\nabla P$ and lamella destruction as a function of $S_{w}$ in the model, and determining the relation between $\mathrm{S}_{\mathrm{w}}{ }^{*}$ and surfactant concentration for this surfactant formulation in our porous medium. 


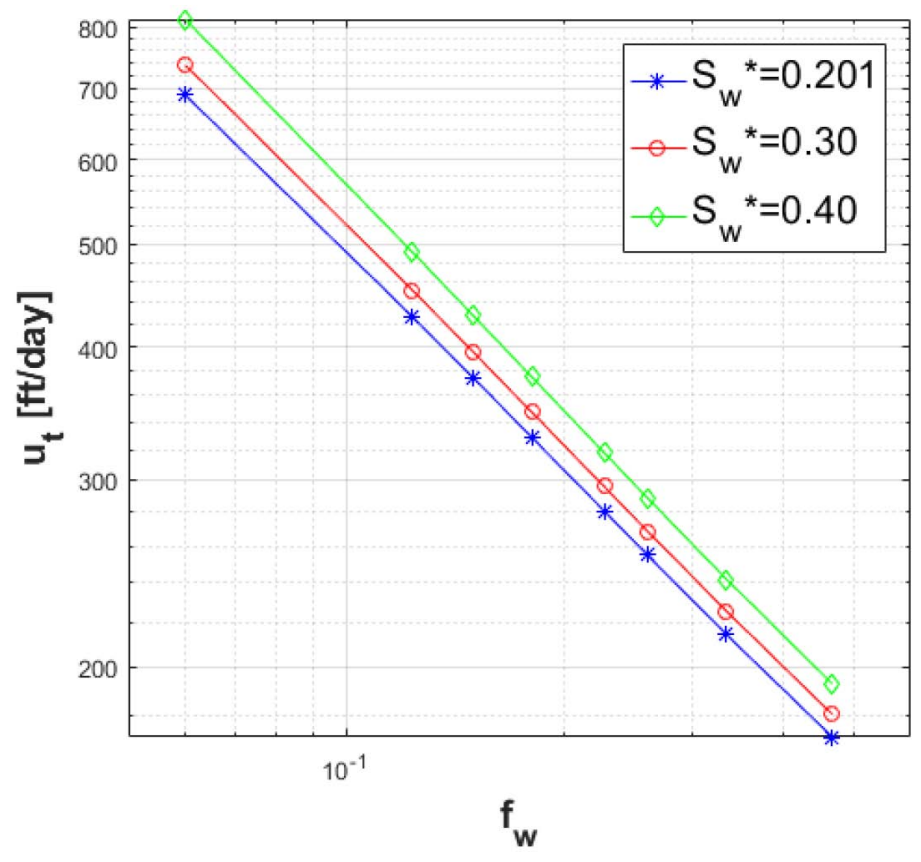

Figure 8-Kam's model's prediction of foam trigger as function of liquid fractional flow $f_{w}$ and limiting liquid saturation $S_{w}{ }^{*}$.

\section{Discussion}

For a given homogeneous porous medium, the trigger velocity or pressure gradient for foam generation depends on the capillary resistance of a lamella to be displaced from a pore throat and subsequent division (Rossen and Gauglitz, 1990). This resistance is of course proportional to the gas-liquid surface tension $\gamma$. Therefore, the critical condition for foam generation depends on surface tension, but this dependence affects foam generation only for surfactant concentrations below the CMC. For surfactant concentrations selected in our experiments, which are far above the $\mathrm{CMC}$, this dependence is absent.

\section{Conclusions}

1. Our data show that the minimum velocity for foam generation in steady flow decreases with increasing surfactant concentration and increasing injected liquid fractional flow $\left(f_{w}\right)$.

2. The impact of surfactant concentration on foam generation that we find in our results is in accord with the prediction of Kam's population-balance model (Kam and Rossen, 2003), where the trigger velocity for foam generation increases with increasing foam quality $\mathrm{f}_{\mathrm{g}}$, and decreases with increasing surfactant concentration $\mathrm{C}_{\mathrm{s}}$ (reflected as $\mathrm{S}_{\mathrm{w}}{ }^{*}$ in Kam's model).

3. Foam generation is closely related to foam propagation. The stability and transport of bubble transport at the leading edge of displacement front requires further investigation. However, our results suggest that foam propagation has a similar dependency on water fractional flow and surfactant concentration: wetter foam and greater surfactant concentration promote the transport of foam, even at surfactant concentrations far above the CMC.

\section{Acknowledgements}

This project is part of the Joint Industry Project (JIP) on foam for EOR. The authors thank and acknowledge both the sponsorship and advice of sponsoring companies and their representatives. Sebastien VincentBonnieu would like to thank Sell Global Solutions International B.V. especially for their review on this article and their permission for publication. In addition, we thank Michiel Slob and Jolanda van Haagen Donker for their technical assistance with our experiments. 


\section{Nomenclature}

$\mathrm{C}_{\mathrm{g}}=$ model parameter (Table A 2)

$\mathrm{C}_{\mathrm{c}}=$ model parameter (Table A 2)

$\mathrm{C}_{\mathrm{s}}=$ surfactant concentration, expressed as [wt $\%$ ]

$\mathrm{f}_{\mathrm{g}}=$ gas fractional flow

$\mathrm{f}_{\mathrm{w}}=$ water fractional flow

$\mathrm{k}=$ permeability, $\left[\mathrm{m}^{2}\right]$

$\mathrm{k}_{\mathrm{rg}}=$ gas relative permeability in absence of foam

$\mathrm{k}_{\mathrm{rw}}=$ water relative permeability

$\mathrm{m}=$ model parameter (Table A 2)

$\mathrm{n}=$ model parameter (Table A 2)

$\mathrm{n}_{\mathrm{f}}=$ foam texture or density, inversely related to bubble size (Eq. A.2), [m-3]

$\nabla P=$ magnitude of pressure gradient

$\Delta P=$ pressure drop across core or section of core

$\nabla P^{\min }=$ minimum pressure gradient required to trigger foam generation

$P_{c}=$ capillary pressure $[\mathrm{Pa}]$

$P_{c}^{*}=$ limiting capillary pressure $[\mathrm{Pa}]$

$S_{w}^{*}=$ limiting water saturation - water saturation at limiting capillary pressure

$\mathrm{S}_{\mathrm{gr}}=$ trapped/residual gas saturation

$\mathrm{S}_{\mathrm{w}}=$ water saturation

$\mathrm{S}_{\mathrm{wc}}=$ connate water saturation (Eq. A 1)

$\mathrm{u}_{\mathrm{g}}=$ gas superficial velocity (Darcy velocity), $[\mathrm{m} / \mathrm{s}]$ in calculations, [ft/D] in figures and texts

$\mathrm{u}_{\mathrm{w}}=$ water superficial velocity (Darcy velocity), $[\mathrm{m} / \mathrm{s}]$ in calculations, $[\mathrm{ft} / \mathrm{D}]$ in figures and texts

$\mathrm{u}_{\mathrm{t}}=$ total superficial velocity (Darcy velocity), $[\mathrm{m} / \mathrm{s}]$ in calculations, $[\mathrm{ft} / \mathrm{D}]$ in figures and texts

$\mathrm{u}_{\mathrm{t}, \mathrm{c}}=$ critical total superficial velocity (Darcy velocity) required for triggering of foam generation, $[\mathrm{m} / \mathrm{s}]$ in calculations, $[\mathrm{ft} / \mathrm{D}]$ in figures and texts

$v_{g}^{\min }=$ minimum gas interstitial velocity required for triggering of foam generation, defined in Figure 2

$\mu_{g}^{0}=$ gas viscosity in absence of foam [Pa・s]

$\mu_{w}=$ water viscosity [Pa・s]

$\phi=$ porosity

$\gamma=$ surface tension (Table A 2), shown here in unit of $[\mathrm{mN} / \mathrm{m}]$

\section{References}

Ashoori, E., Marchesin, D., and Rossen, W. R. 2011. Roles of Transient and Local Equilibrium Foam Behaviour in Porous Media: Traveling Wave. Colloids Surf. A 337 (1-3): 228-242.

Apaydin, O. G., and Kovscek, A. R. 2001. Surfactant Concentration and End Effects on Foam Flow in Porous Media. Transport in Porous Media 43:511-536.

Baghdikian, S., and Handy, L. L. 1990. Flow of Foaming Solutions in Porous Media. Modification of Chemical and Physical Factors in Steam-flood to Increase Heavy Oil Recovery. Y. C. Yortsos, U. S. Dept. Energy Ann. Rep., DOE/ BC/14126-14, Bartlesville, OK.

Chou, S. I. 1991. Conditions for Generating Foam in Porous Media, in: Paper SPE 22628 Presented at the SPE Annual Technical Conference and Exhibition, Dallas, TX.

Douglas, J., Blair, P. M. and Wagner, R. J. 1958. Calculation of linear waterflood behaviour including the effect of capillary pressure. Trans. AIME 214, 96-102. 
Falls, A. H., Hirasaki, G. J., Patzek, T. W., Gauglitz, P. A., Miller, D. D., Ratulowski, J. 1988. Development of a mechanistic foam simulator: the population balance and generation by snap-off. SPERE 3(3), 884-892.

Friedmann, F., Chen, W. H., Gauglitz, P. A. 1991. Experimental and simulation study of high-temperature foam displacement in porous media. SPERE 6(1), 37-45.

Friedmann, F., Smith, M. E., Guice, W. R., Gump, J. M., Nelson, D. G. 1994. Steam-foam mechanistic field trial in the midway-sunset field. SPERE 9(4), 297-304.

Gauglitz, P. A., and Radke, C. J. 1989. The Dynamic of Liquid Film Breakup in Constricted Cylindrical Capillaries. Colloid and Interface Science 134, No. 1.

Gauglitz, P. A., Friedmann, F., Kam, S. I., and Rossen, W. R. 2002. Foam Generation in Homogeneous Porous Media. Chem. Eng. Sci. 57, 4037-4052.

Huh, D. G., and Handy, L. L. 1989. Comparison of Steady- and Unsteady-State Flow of Gas and Foaming Solution in Porous Media. Soc. Pet. Eng. Reservoir Eng. 4, 77.

Jacquard, P. 1991. Improved Oil Recovery in the Global Energy Perspective. 6th European Symposium. Improved Oil Recovery (Stavanger, May 21-23), Plenary Conference.

Jones, S. A., Laskaris, G., Vincent-Bonnieu, S., Farajzadeh, R., and Rossen, W. R. 2016. Effect of Surfactant Concentration on Foam: From Coreflood Experiments to Implicit-Texture Foam-Model Parameters. J. Ind. \& Eng. Chem. 37, 268-276.

Kam, S. I., and Rossen, W. R. 2003. A Model for Foam Generation in Homogeneous Media. SPE J. 8(4): 417-425. SPE-87334-PA.

Kam, S. I. 2008. Improved Mechanistic Foam Simulation with Foam Catastrophe Theory. Colloids Surf., A 318 (1-3): 62-77.

Kovscek, A. R., Patzek, T. W., and Radke, C. J. 1995. A Mechanistic Population Balance Model for Transient and SteadyState Foam Flow in Boise Sandstone. Chem. Eng. Sci. 50 (23): 3783-3799.

Khatib, Z. R., Hirasaki, G. J., and Falls, A. H. 1988. Effects of Capillary Pressure on Coalescence and Phase Mobilities in Foams Flowing Through Porous Media. SPE Res Eng 3 (3): 919-926. SPE-15442-PA.

Kyte, J. R. Rapoport, L. A. 1958. Linear Waterflood Behaviour and End Effects in Water-Wet Porous Media. Petroleum Trans. AIME, 213, 47-50.

Lake, L. W., Johns, R. T., Rossen, W. R., and Pope, G. A. 2014. Fundamentals of Enhanced Oil Recovery. Soc. Pet. Eng, ISBN 978-1-61399-407-8.

Ma, K., Lopez-Salinas, J. L., Puerto, M. C., Miller, C. A., Biswal, S. L., and Hirasaki, G. J. 2013. Estimation of Parameters for the Simulation of Foam Flow Through Porous Media. Part 1: The Dry-out Effect. Energy \& Fuels 27 (5): 2363-2375.

Moritis, G. 1990. CO2 and HC Injection Lead EOR Production Increase. Oil \& Gas J.: 49-81.

Nguyen, Q. P., Currie, P. K., and Zitha, P. L. J. 2003. Determination of Foam Induced Fluid Partitioning in Porous Media Using X-Ray Computed Tomography. Paper SPE80245 presented at the International Symposium on Oilfield Chemistry, Houston, TX, USA, 5-8.

Perkins F. M. 1957. An Investigation of the Role of Capillary Forces in Laboratory Water Floods. Trans. AIME, $207,49-51$.

Ransohoff, T. C., and Radke, C. J. 1988. Mechanics of Foam Generation in Glass Bead Packs. Soc. Pet. Eng. Reservoir Eng., 3, 573.

Rossen, W. R., and Gauglitz, P. A. 1990. Percolation Theory of Creation and Mobilization of Foams in Porous Media. AI Chem Eng. J. Vol. 36, No. 8.

Rossen, W. R. 1996. Foams in Enhanced Oil Recovery. In: Prud'homme, R. K., Khan, S. (Eds.), Foams: Theory Measurement and Applications. Marcel Dekker, New York City.

Simjoo, M., and Zitha, P. L. J. 2013. Effects of Oil on Foam Generation and Propagation in Porous Media. Paper SPE 165271 presented at the SPE Enhanced Oil Recovery Conference, Kuala Lumpur, Malaysia, 2-4.

Wonnacott, T. H., and Wonnacott, R. J., Introductory Statistics for Business and Economics, John Wiley and Sons., 1972.

Zhou, Z., and Rossen, W. R. 1995. Applying Fractional-Flow Theory to Foam Processes at the "Limiting Capillary Pressure". Society of Petroleum Engineers. doi:10.2118/24180-PA. 


\section{Appendix}

Table A 1-Foam qualities and surfactant concentrations selected for foam-generation experiments.

\begin{tabular}{|cc|cccccccc|}
\hline & & \multicolumn{7}{|c|}{ Foam Quality f $f_{g}[\%]$} \\
\hline $\mathrm{Cs}$ & 0.1 & 94.01 & 87.67 & 85.04 & 81.98 & 77.34 & 73.98 & 66.67 & 53.22 \\
{$[\mathrm{wt} \%]$} & 0.3 & 94.01 & 87.67 & 85.04 & 81.98 & 77.34 & 73.98 & 66.67 & - \\
& 0.5 & 94.01 & 87.67 & 85.04 & 81.98 & 77.34 & 73.98 & 66.67 & - \\
\hline
\end{tabular}

$$
\begin{gathered}
k_{r g}=\left(\frac{1-S_{w}-S_{g r}}{1-S_{w c}-S_{g r}}\right)^{2.2868} \\
k_{r w}=0.7888\left(\frac{S_{w}-S_{w c}}{1-S_{w c}-S_{g r}}\right)^{1.9575}
\end{gathered}
$$

Relative permeability functions used in Kam's model (values from Kam \& Rossen, 2003).

Table A 2-Parameter values used in Kam's model for prediction of

\begin{tabular}{|c|c|c|c|}
\hline \multicolumn{4}{|c|}{$\begin{array}{l}\text { Parameter values used in Kam's model for } \\
\text { prediction of triggering superficial velocities }\end{array}$} \\
\hline \multicolumn{2}{|c|}{ Foam Parameters } & \multicolumn{2}{|c|}{ Other Parameters } \\
\hline $\mathrm{C}_{\mathrm{g}} / \mathrm{C}_{\mathrm{c}}$ & $1 \times 10^{-13}$ & $S_{w}^{*}$ & $\begin{array}{c}{[0.201,0.30} \\
0.40]\end{array}$ \\
\hline M & 4.4 & $\mathrm{~K}\left[\mathrm{~m}^{2}\right]$ & $7.1 \times 10^{-12}$ \\
\hline $\mathrm{N}$ & 0.85 & $\Phi$ & 0.199 \\
\hline \multirow[t]{4}{*}{$C_{f}$} & $1 \times 10^{-14}$ & $\mu_{w}[\mathrm{~Pa} \bullet \mathrm{s}]$ & 0.001 \\
\hline & & $\boldsymbol{\mu}_{\boldsymbol{g}}^{\mathbf{0}}[\mathrm{Pa} \bullet \mathrm{s}]$ & 0.00002 \\
\hline & & $S_{w c}$ & 0.2 \\
\hline & & $S_{g r}$ & 0.1 \\
\hline
\end{tabular}
trigger velocity for foam generation (values from Kam \& Rossen, 2003). 